\title{
VIRTUAL SCREENING OF POTENTIAL INHIBITORS FROM HERBS FOR THE TREATMENT OF BREAST CANCER
}

\author{
FAIZY KHAN, SHANTANU BAFNA, TANU GUPTA, ARNOLD EMERSON I*
}

\section{Department of Biotechnology, School of Bio Sciences and Technology, VIT University, Vellore, India. Email: i_arnoldemerson@yahoo.com}

Received: 30 August 2016, Revised and Accepted: 03 January 2017

\section{ABSTRACT}

Objectives: Cancer is a disease which results in uncontrollable abnormal cells division and destruction of body tissues. Breast cancer occurs when malignant tumors develop in the breast. Breast cancer is the second leading cause of death among women. To study the role of herbs used in the treatment for breast cancer. To investigate the anti-breast cancer activity of compounds present on most common herbs and to analyse their interaction with amino acids in the active sites.

Methods: Complementary and alternative medicine is often used for curing cancer mainly the breast cancer. Also certain studies support the benefits of herbal medicines over others among Complementary and alternative medicine. Herbal treatments are more popular due to less complications and more safety. We selected a dataset of 38 compounds and performed virtual screening to identify the potential inhibitor against the known protein target BRCA1 involved in breast cancer using AutoDock4 as docking software. The binding site analyses were carried out using Discovery studio.

Results: From our study, we deduced that cimigenol (black cohosh) and glycyrrhetinic acid (licorice) were found to have the highest affinity with the target protein. The amino acid interactions with the top five compounds were also analysed.

Conclusion: During the course of our research we explored over common herbs used globally in treatment for breast cancer. Virtual screening was performed using AutoDock to search ligands to identify those structures which are most likely to bind to the protein. The high affinity compounds can bind more efficiently to the BRCA1 receptor and, hence, has potential to emerge as lead compound in the treatment of breast cancer.

Keywords: Protein, Ligands, AutoDock, Virtual screening, Visualization, BRCA1.

(C) 2017 The Authors. Published by Innovare Academic Sciences Pvt Ltd. This is an open access article under the CC BY license (http://creativecommons. org/licenses/by/4. 0/) DOI: http://dx.doi.org/10.22159/ajpcr.2017.v10i4.14959

\section{INTRODUCTION}

Cancer is a state when body cells begin to grow out of control. Cells in any part of the body can become cancerous and can spread to other areas. Germline mutations in a gene on chromosome $17 q$ known as BRCA1 are responsible for a large proportion of inherited predispositions to breast and ovarian cancer [1]. Breast cancer is a fatal tumor; a cluster of cancerous cells that can enter adjoining tissues or can extent to far away areas of the body. These cells grow by disrupting away from the original tumor and then go into the blood vessels which branch into tissues within the body. The disease occurs majorly in women, but men can get it, too. Breast cancer generally begins in the inner lining of milk ducts that supply them with milk. A breast cancer of the lobules is known as lobular carcinoma while that of the ducts is called ductal carcinoma. It accounts for $16 \%$ of all female cancers and $22.9 \%$ of invasive cancers in women, also $18.2 \%$ of all cancer deaths worldwide, including both males and females, are from breast cancer [2].

Several techniques and clinical methods which include relaxation tactics such as - massage and herbal remedies are now recognized as essential part of alternative medicine. These practices suggest that the uses of alternative medicines are used by the section of population which have a particular education level and reached that level of income.

Most of the patients in the United Kingdom use various supplements to enhance their fitness or provide a good start to their immune system. Dietary therapies were often practiced which had special diet plans-low fat diet, eating only vegetarian food, freshly prepared meals, fruits, and whole grains. Herbal remedies include mixtures of herbs and plants, use of garlic oil for hypertension, Echinacea for proper functioning of the immune system. Many relaxation techniques that calm the body by lowering blood pressure and pulse rates are often used to heal the tension in the body. Meditation or spiritual healing which connects one to a higher power is also believed to calm the body tension.

Virtual screening is a method used in drug designing to generate or search large databases of small molecules (or ligands) to bind to a particular site in a protein or drug molecule [3]. It encompasses a variety of computational techniques, helping chemists to reduce a huge virtual library to a more manageable size [4]. In this technique, the crystal structure of the protein molecule was taken from the protein data bank [5]. All structures of the ligand molecules included in the database for testing whether the molecule binds or not were also taken from the protein data bank. These molecules were then docked using the AutoDock Software. Based on the binding energies and the three-dimensional (3D) structures generated the ligand molecules were sorted. These structures were then used to find out which of the structures that were docked in the given protein structure were stable (Based on hydrophilic and hydrophobic amino acids present in the structure and number of hydrogen bonds in the structure). Virtual screening, especially receptor-based virtual screening, has emerged as a reliable, inexpensive and accessible method for identifying leads [6].

In our research work, we installed the required Software's (AutoDock 4.2.3-i86 Linux 2.tar.gz, MGL Tools-1.5.6, and Tcsh) and then used python script. The protein and ligand were selected. Then series of steps were carried out to prepare a library containing ligand files and corresponding AutoGrid and AutoDock parameters files for library. After which AutoGrid was used to calculate maps also to launch AutoDock calculations for each ligand followed by two analyses step in which we extracted and evaluated results through which we got the sorted list of ligands according to the energy to bind with the protein [7]. Further analysis of result was carried out and the best conformation 
of every ligand was selected. After which these ligands and the protein were visualized together as a complex in discovery studio to get their two-dimensional (2D) image.

\section{METHODS}

\section{Protein structure}

Mutations in BRCA1 and BRCA2 confer high risks of breast cancer [2]. From gene card database [8], proteins related to BRCA1 gene were obtained from which the protein with the best resolution (lowest) was selected. The X-ray crystal structure of the protein BRCA1 at $1.75^{\circ} \mathrm{A}$ resolution (PDB ID: 4IGK), were retrieved from protein data bank [9]. AutoDock expects that the input protein has polar hydrogen and that all the water molecules should be removed. Hence before protein preparation process, all the water molecules along with the heteromolecule attached with the structures were removed from the original crystal structure of 4IGK. Polar hydrogen atoms and Kollman charges were added to the protein structure.

\section{Herbal compounds for breast cancer}

The most common herbs (eleven) used in treatment for breast cancer were taken. Their composition was selected from "examine website database" (Table 1) [10]. Then the sdf structure of the components of these herbs was downloaded from Pubchem database [11]. These sdf files were converted to $\mathrm{mol}^{2}$ format using Babel Software [12] and then we applied Lipinski rule of five to further filter the molecules as shown in Table 2.

\section{Virtual screening using AutoDock}

AutoDock is docking software structured in a way to predict the binding of a substrate to a receptor molecule and virtual screening is a technique used for identifying assuring compounds to bind to a target molecule with a known structure [13]. It automatically calculates the grid map and clusters the results in a way transparent to the user [14]. We have used the AutoDock version 4.2.3. This software has two main functions: First is AutoDock, which performs docking of a ligand molecule to a specific receptor site in the protein molecule [15]. Second is AutoGrid, for precalculating the grids or the specific locations where a particular ligand molecule bind in a protein molecule [16]. This software is often used for structure-based drug design as it helps in predicting atomic affinity grids in the molecule.

The ligand molecule is provided with a set of binding sites in the protein molecule which is found out by trying various conformations depending on the structure of the ligand and translations of the ligand molecule. The energy of the ligand-protein molecule is also calculated at different binding sites.

With the time, virtual screening has become an accepted tool in drug discovery. It has been successfully applied in a number of programs, in particular, at the lead discovery stage, where high-throughput molecular docking can play an important role [17]. Now, the virtual screening is carried out by creating a folder directory called "virtual screening" containing sub folders called "ligands," etc., in the "ligand" folder ligand files are set up. The ligand database consists of sdf files that are downloaded from the Protein Data Bank, which is then converted to $\mathrm{mol}^{2}$ file format using babel software. Then, the preparing of ligand was done in which $\mathrm{mol}^{2}$ files prepared in the previous step are then processed to ".pdbqt" file format using AutoDock software. These molecule files must be supported by AutoDock, following a given set of atom types. This set includes: United-atom aliphatic carbons, cycles having aromatic carbons, polar hydrogens, hydrogen-bonding nitrogens and directionally hydrogen-bonding oxygens among others, each with a partial charge. To determine the covering set of atom types a 3D lattice of equally spaced points placed over the ligand molecule and the location or active site in the protein molecule. AutoGrid software is used to calculate grid map for each constituent atom present in the ligand to be docked in the protein molecule. Now the receptor is prepared by saving the ligand files in pdbqt format. In pdbqt, "q" represents the charge and " $\mathrm{t}$ " is the type of AutoDock. Polar hydrogen atoms are added to the molecule and assigned a gasteiger partial charge. Further, the AutoGrid parameter files for the library is prepared. Dimensions are provided to grid box which completely surrounds the protein and ligand molecule. AutoGrid process the ligand molecule conformations that can be docked in the protein molecule on the 3D grid point in the grid box. All the energies of different sites of docking are noted by the software. Now to calculate atomic affinity maps for a ligand library, AutoGrid was used. The "ligand" folder contains the map files and ligand.pdbqt file formats. After AutoGrid takes place each of the ligand atoms will have a ".pdbqt" file and a docking parameter file with ".dpf" format. The docking directories and parameter files for each ligand in a library is prepared and AutoDock is run to find the docking energy and various docked conformations of the molecule. Finally, analysis is

Table 1: Herbal compounds with PubChem ID

\begin{tabular}{|c|c|c|c|}
\hline S. $\mathbf{N}$ & Herb name & Constituent name & PubChem ID \\
\hline 1 & Echinacea & Caffeic acid & 689043 \\
\hline 2 & Echinacea & Chicoric acid & 5281764 \\
\hline 3 & Echinacea & Echinacoside & 5281771 \\
\hline 4 & Echinacea & Luteolin & 5280637 \\
\hline 5 & Echinacea & Rutoside & 5280805 \\
\hline 6 & Licorice & Formononetin & 5280378 \\
\hline 7 & Licorice & Glabridin & 124052 \\
\hline 8 & Licorice & Glycyrrhetinic acid & 10114 \\
\hline 9 & Licorice & Glycyrrhizic acid & 14982 \\
\hline 10 & Licorice & Liquiritigenin & 114829 \\
\hline 11 & Cat's claw & Isopteropodine & 9885603 \\
\hline 12 & Cat's claw & Lyaloside & 11092621 \\
\hline 13 & Cat's claw & Mitraphylline & 94160 \\
\hline 14 & Cat's claw & Tomentside B & 21629530 \\
\hline 15 & Cat's claw & Uncaric acid & 71448960 \\
\hline 16 & Cat's claw & Uncarinec & 98363 \\
\hline 17 & Garlic & Diallyl Disulfide & 16590 \\
\hline 18 & Garlic & Dithiins & 12044565 \\
\hline 19 & Garlic & S-allyl cysteine & 9793905 \\
\hline 20 & Flaxseed & Linamarin & 11128 \\
\hline 21 & Flaxseed & Linustatin & 119301 \\
\hline 22 & Flaxseed & Lotaustralin & 441467 \\
\hline 23 & Flaxseed & Neolinustatin & 119533 \\
\hline 24 & Flaxseed & $\begin{array}{l}\text { Secoisolariciresinol } \\
\text { Diglucoside }\end{array}$ & 9917980 \\
\hline 25 & Turmeric & Bisdemethoxycurcumin & 5315472 \\
\hline 26 & Turmeric & Calebin-A & 637429 \\
\hline 27 & Turmeric & Curcumin & 969516 \\
\hline 28 & Turmeric & Cyclocurcumin & 69879809 \\
\hline 29 & Turmeric & Demethoxycurcumin & 5469424 \\
\hline 30 & Burdock & Arctigenin & 64981 \\
\hline 31 & Burdock & Chalcone & 637760 \\
\hline 32 & Burdock & Flavanol & 253959 \\
\hline 33 & Burdock & Flavanone & 10251 \\
\hline 34 & Burdock & Flavone & 10680 \\
\hline 35 & Burdock & Phlorotannin A & 73162010 \\
\hline 36 & Burdock & Proanthocyanidin & 108065 \\
\hline 37 & Carotenoids & Capsanthin & 5281228 \\
\hline 38 & Carotenoids & Capsorubin & 5281229 \\
\hline 39 & Green tea & Aminobutyric acid & 119 \\
\hline 40 & Green tea & Catechins & 9064 \\
\hline 41 & Green tea & Epicatechin & 72276 \\
\hline 42 & Green tea & Epigallocatechin & 72277 \\
\hline 43 & Green tea & Theanine & 439378 \\
\hline 44 & Ginseng & Ginsenoside Rg3 & 9918693 \\
\hline 45 & Ginseng & Ginsenosides & 3086007 \\
\hline 46 & Ginseng & Panaxtriol & 73599 \\
\hline 47 & Ginseng & Ginsenoside Rb1 & 9898279 \\
\hline 48 & Ginseng & Ginsenoside RG1 & 441923 \\
\hline 49 & Black cohosh & Actein & 21594792 \\
\hline 50 & Black cohosh & Cimicifugoside & 441913 \\
\hline 51 & Black cohosh & Cimigenol & 16020000 \\
\hline 52 & Black cohosh & Salsolinol & 91588 \\
\hline
\end{tabular}


Table 2: Binding energies for the docked herbal compounds

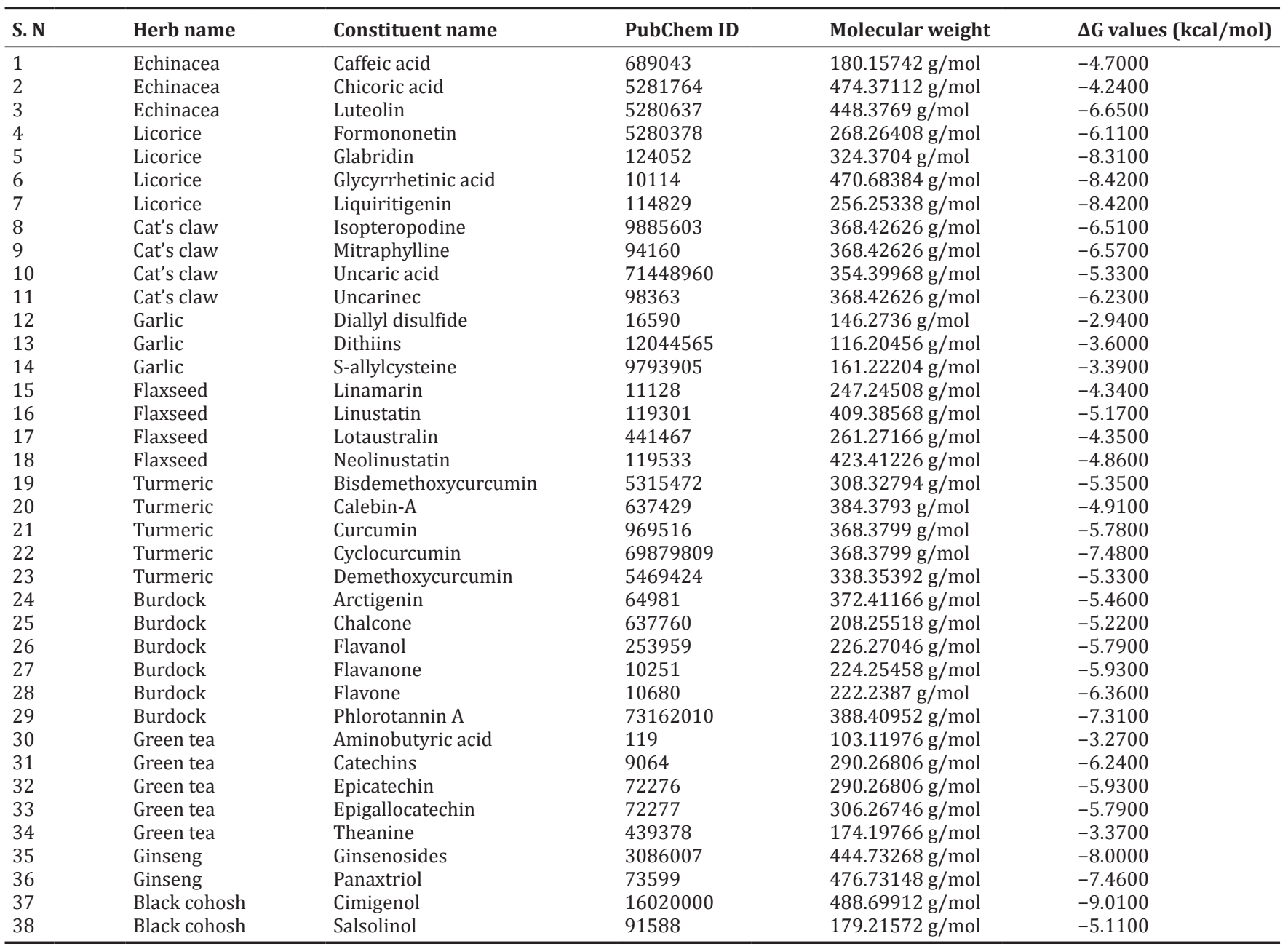

done using AutoDock tools. The results of docking are studied based on structural and conformational similarity and binding sites. Best docked conformation is found by a list provided with molecules sorted by lowest energy of docking [18].

\section{Discovery studio}

Discovery studio is a suite of software for simulating small molecule and macromolecule systems. It is developed and distributed by accelrys [19]. Discovery studio provides software applications covering the areas such as simulations, ligand design, and structure-based design. Discovery studio is used for the visualization of interaction between protein and ligand. To view the receptor ligand interaction the complex was opened and its 2D diagram was obtained.

\section{RESULTS}

After performing virtual screening using AutoDock, we obtained the sorted list of ligands based on their binding energy with the receptor (4IGK) which was further used to find the optimal conformation of the ligand to be used with the receptor [18].

\section{Binding site analysis}

1. Cimigenol compound

The 2D crystal structure of complex was acquired from discovery studio after performing virtual screening using AutoDock. The residues bounded to the active site are TYR1845, GLN1846, CYS1847, ILE1760, LYS1759, ARG1758, SER1755, ARG1751, and GLU1754. The binding energy of this complex was found to be $-9.0100 \mathrm{kcal} / \mathrm{mol}$. Among the residues the one which is hydrophobic is CYS1847 while GLU1754, GLN1846, ARG1751, and ARG1758 are hydrophilic in nature. TYR1845 is aromatic in nature where as ILE1760 is nonpolar as well as aliphatic in nature. TYR1845, GLN1846, LYS1759, SER1755, and GLU1754 have van der Waals interactions with the Ligand (black cohosh cimigenol 2D) on the other hand ARG1751, ARG1758, ILE1760, and CYS1847 are singly bonded. Studies revealed that cimigenol and related compounds were screened as potential antitumor promoters by using the in vitro short-term 12-0-tetradecanoylphorbol-13-acetate (TPA)-induced EpsteinBarr virus early antigen activation assay [20].

2. Glycyrrhetinic acid

By the use of discovery studio, we acquired 2D crystal structure of protein-ligand complex after executing virtual screening using AutoDock. The binding energy of this complex was found to be $-8.4200 \mathrm{kcal} / \mathrm{mol}$. The residues bounding the active site are TYR1845, GLN1846, CYS1847, ILE1760, LYS1759, ARG1758, SER1755, ARG1751, and ARG1762. Among these residues, the one which is hydrophobic is CYS1847 while GLN1846, ARG1751, ARG1762, and ARG1758 are hydrophilic in nature. TYR1845 is aromatic in nature whereas ILE1760 is nonpolar as well as aliphatic in nature. TYR1845, GLN1846, and LYS1759 have van der Waals interactions with the Ligand (licorice glycyrrhetinic acid) on the other hand ARG1762, ARG1751, ILE1760, and CYS1847 are singly bonded. ARG1758 has unfavorable DonorDonor bond and SER1755 has conventional hydrogen bond with the ligand. Recent study found that this compound impairs the p38 MAPK-AP1 signaling axis which accelerates the repression of breast cancer cell invasion [21]. 


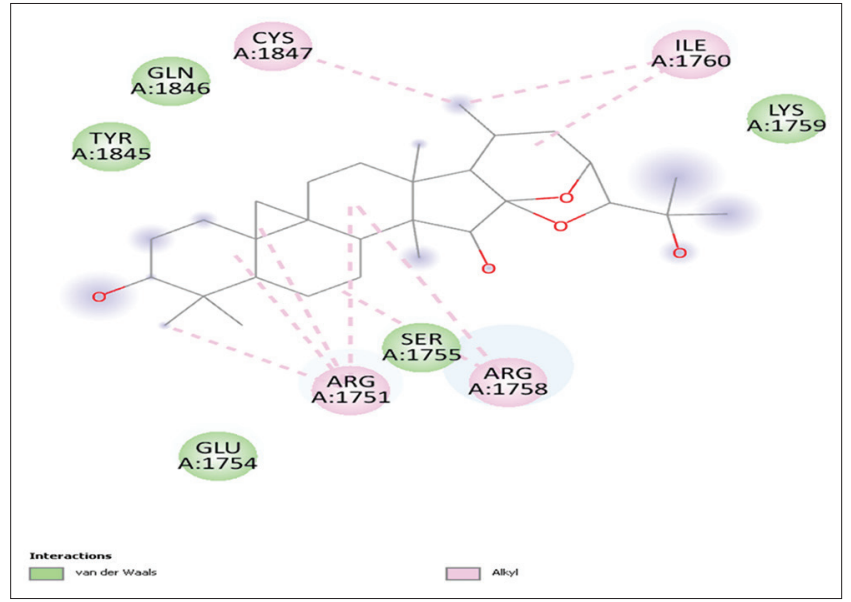

Fig. 1: Amino acid interaction of BRCA1 protein complex with cimigenol

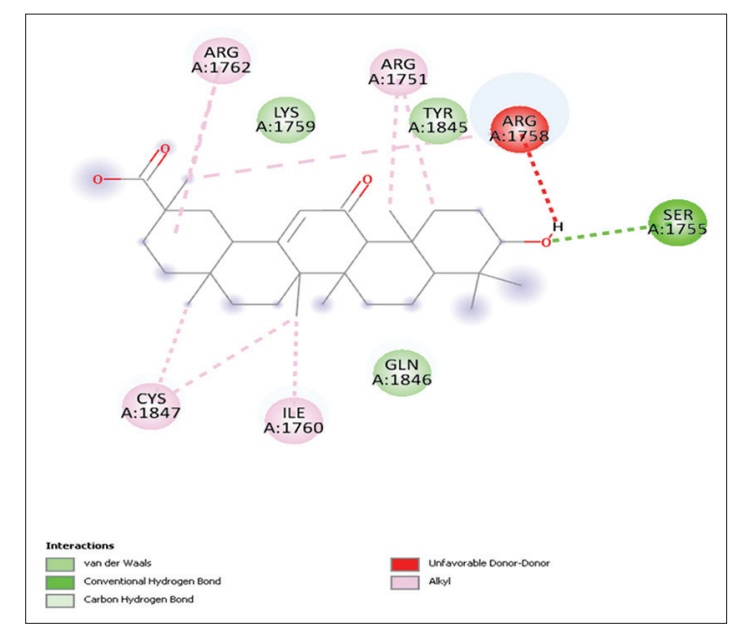

Fig. 2: Amino acid interaction of BRCA1 protein complex with glycyrrhetinic acid

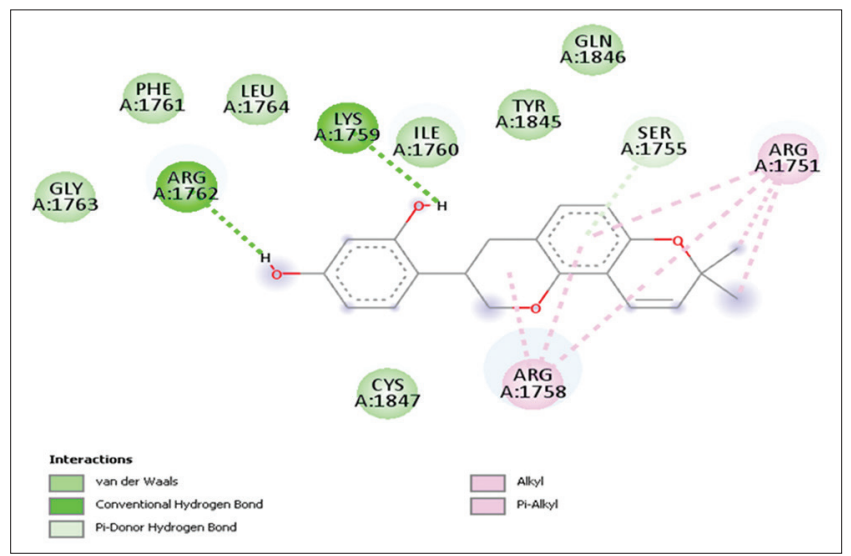

Fig. 3: Amino acid interaction of BRCA1 protein complex with glabridin

3. Glabridin compound

Discovery studio was used to obtain the 2D crystal structure of protein-ligand complex after executing virtual screening using AutoDock. The residues bounding the active site are TYR1845, GLN1846, CYS1847, ILE1760, LYS1759, ARG1758, SER1755, ARG1751, ARG1762, GLY1763, PHE1761, and LEU1764. The complex has a binding energy of $-8.3100 \mathrm{kcal} / \mathrm{mol}$. Amid

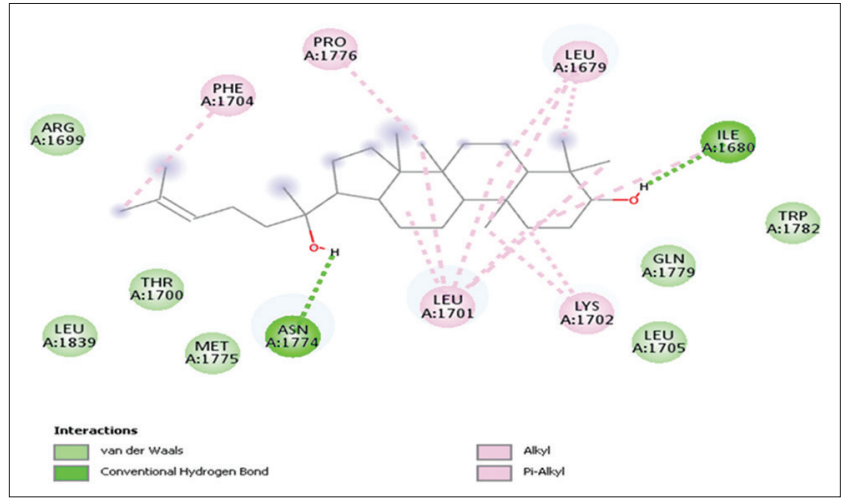

Fig. 4: Amino acid interaction of BRCA1 protein complex with ginsenosides

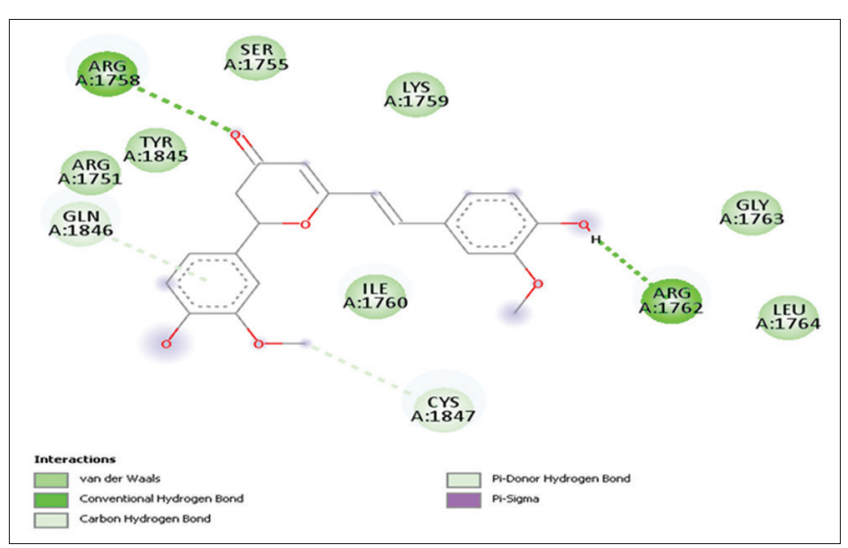

Fig. 5: Amino acid interaction of BRCA1 protein complex with cyclocurcumin

the residues the one which is hydrophobic is CYS1845 while GLN1846, ARG1751, ARG1762, and ARG1758 are hydrophilic in nature. TYR1845 and PHE1761 are aromatic in nature, whereas ILE1760, GLY1763, and LEU1764 are nonpolar as well as aliphatic in nature. TYR1845, GLN1846, CYS1847, ILE1760 LEU1764, PHE1761, and GLY1763 have van der Waals interactions with the ligand (licorice glabridin) on the other hand ARG1751 and ARG1758 are singly bonded and LYS1759 and ARG1762 has conventional hydrogen bond with the ligand. Furthermore, SER1755 has Pi-Donor hydrogen bond with the ligand. In mouse xenograft models, this glabridin compound was found to attenuated the tumor growth, mesenchymal characteristics, and cancer stem cells-like properties via demethylation-activated miR-148a [22].

4. Ginsenosides compound

By executing virtual screening using AutoDock in discovery studio, the 2D crystal structure of protein-ligand complex was obtained as shown in Fig. 4. The binding energy of this complex was found to be $-8.0000 \mathrm{kcal} / \mathrm{mol}$. The residues bounding the active site are ARG1699, PHE1704, PR01776, LEU1679, ILE1680, TRP1782, GLN1779, LEU1705, LYS1702, LEU1701, ASN1774, MET1775, THR1700, and LEU1839. Among the residues the one which are hydrophobic are PHE1704, MET1775 and PR01776 while GLN1779, ASN1774 and ARG1699 are hydrophilic in nature. PHE1704 and TRP1782 are aromatic in nature whereas ILE1680, and PR01776 are aliphatic in nature. ARG1699, THR1700, LEU1839, MET1755, GLN1779, TRP1782, and LEU1705 have van der Waals interactions with the ligand (ginseng ginsenosides) on the other hand PHE1704, PR01776, LEU1679, LEU1701, and LYS1702 are singly bonded and ASN1774 and ILE1680 has conventional hydrogen bond with the ligand. Ginseng root is one of the most commonly 
used herbal medicines in the United States and East Asia for its multi-pharmaceutical functions [23,24]. Intake of ginseng root was reported to associate with reduced risk of various types of cancer in a case-control study conducted in Korea [25].

5. Cyclocurcumin

Discovery studio helped in performing virtual screening using AutoDock through which the 2D crystal structure of complex was gained. The residues bounded to the active site are TYR1845, GLN1846 (Fig. 5), CYS1847, ILE1760, LYS1759, ARG1758, SER1755, ARG1751, LEU1764, ARG1762, and GLY1763. The binding energy of this complex was found to be $-7.4800 \mathrm{kcal} / \mathrm{mol}$. Among the residues, the one which is hydrophobic is CYS1847 while GLN1846, ARG1751, ARG1762, and ARG1758 are hydrophilic in nature. TYR1845 is aromatic in nature where as ILE1760, LEU1764, and GLY1763 are nonpolar as well as aliphatic in nature. TYR1845, ARG1751, ILE1760, LEU1764, LYS1759, SER1755, and GLY1763 have van der Waals interactions with the ligand (turmeric cyclocurcumin) on the other hand ARG1758 and ARG1762 have conventional hydrogen bonds with the ligand. Pi donor hydrogen bond is also present. CYS1847 and GLN1846 constitute this bond. Curcumin has been shown to exhibit antioxidant, anti-inflammatory, antimicrobial, and anticarcinogenic activities. Several studies suggested that cyclocurcumin compound were found to be inhibiting proliferation of breast cancer cells [26-28].

\section{DISCUSSION}

The herbal compounds from black cohosh and licorice were shown to be a better affinity towards breast cancer target protein. Black cohosh has a history of traditional use among Native Americans for the treatment of a variety of disorders, including various conditions unique to women such as amenorrhea and menopause. Contemporary uses of black cohosh are primarily geared toward the treatment of symptoms of menopause, such as hot flashes, and menopausal anxiety and depression [29]. Black cohosh is among the most frequently cited agent being used by breast cancer patients during their radiotherapy and chemotherapy. Black cohosh (Cimicifuga racemosa) is a shrub like plant commonly seen in the eastern forests of North America [30]. Black cohosh is being utilized by women who have been recommended to avoid HRT (Hormonal Replacement Therapy) by their doctors, who are at high risk for breast cancer or who have discontinued HRT after a diagnosis of breast cancer [30]. Glycyrrhetinic acid (GA), a major component of Radix Glycyrrhiza, is actually a significantly more potent agent to suppress invasion than cell survival[31]

Computational Biology and bioinformatics have the potential not only of speeding up the drug discovery process thus reducing the costs, but also of changing the way drugs are designed. Rational Drug Design (RDD) helps to facilitate and speedup the drug designing process, which involves variety of methods to identify novel compounds. One such method is the docking of the drug molecule with the receptor (target). The site of drug action, which is ultimately responsible for the pharmaceutical effect, is a receptor [32]. Docking is the process by which two molecules fit together in 3D space.

\section{CONCLUSION}

In our study, we have analyzed the interaction of different ligands with the receptor protein taken from the BRCA1 gene. We performed virtual screening using AutoDock to find the best conformation of each of these ligands and obtained the conformation having the best binding energy with receptor. This knowledge of the binding energy of different ligands with the receptor can help in the production of various complementary and alternative medicines for breast cancer which can help in curing it with less side effects and complications to the patient. From the research, we deduced that cimigenol; component of the herb black cohosh and glycyrrhetinic acid; component of the herb licorice have the highest affinity to bind with the protein. Thus these two herbs can prove to be most effective in curing breast cancer. Therefore, the future research can be extended to completely utilize the potential of this concept and bring about further novel applications which proved to be major scientific aspiration.

\section{ACKNOWLEDGMENTS}

Authors would like to thank VIT University, Vellore, for providing the laboratory resources during the course of our study.

\section{REFERENCES}

1. Ford D, Easton DF, Bishop DT, Narod SA, Goldgar DE. Risks of cancer in BRCA1-mutation carriers. Breast Cancer Linkage Consortium. Lancet 1994;343(8899):692-5.

2. Antoniou AC, Spurdle AB, Sinilnikova OM, Healey S, Pooley KA, Schmutzler RK, et al. Common breast cancer-predisposition alleles are associated with breast cancer risk in BRCA1 and BRCA2 mutation carriers. Am J Hum Genet 2008;82(4):937-48.

3. Schneider G, Bohm HJ. Virtual screening and fast automated docking methods. Drug Discov Today 2002;7(1):64-70.

4. Walters WP, Stahl MT, Murcko MA. Virtual screening - An overview. Drug Discov Today 1998;3(4):160-78.

5. Bernstein FC, Koetzle TF, Williams GJ, Meyer EF Jr, Brice MD, Rodgers JR, et al. The Protein Data Bank. A computer-based archival file for macromolecular structures. Eur J Biochem 1977;80(2):319-24.

6. Lyne PD. Structure-based virtual screening: An overview. Drug Discov Today 2002;7(20):1047-55

7. Olsak M, Filipovic J, Prokop M. FastGrid--The accelerated AutoGrid potential maps generation for molecular docking. Comput Inform 2012;29(6):1325-36.

8. Rebhan M, Chalifa-Caspi V, Prilusky J, Lancet D. GeneCards: Integrating information about genes, proteins and diseases. Trends Genet 1997;13(4):163.

9. Berman HM, Battistuz T, Bhat TN, Bluhm WF, Bourne PE, Burkhardt $\mathrm{K}$, et al. The protein data bank. Acta Crystallogr D Biol Crystallogr 2002;58(6):899-907

10. Available from: http://www. examine.com

11. Wang Y, Xiao J, Suzek TO, Zhang J, Wang J, Zhou Z, et al. PubChem's BioAssay Database. Nucleic Acids Res 2012;40:D400-12.

12. O'Boyle NM, Banck M, James CA, Morley C, Vandermeersch T, Hutchison GR. Open Babel: An open chemical toolbox. J Cheminform 2011:3:33.

13. Cosconati S, Forli S, Perryman AL, Harris R, Goodsell DS, Olson AJ. Virtual screening with AutoDock: Theory and practice. Expert Opin Drug Discov 2010;5(6):597-607.

14. Trott O, Olson AJ. AutoDock Vina: Improving the speed and accuracy of docking with a new scoring function, efficient optimization, and multithreading. J Comput Chem 2010;31(2):455-61.

15. Park H, Lee J, Lee S. Critical assessment of the automated AutoDock as a new docking tool for virtual screening. Proteins Struct Funct Bioinform 2006;65(3):549-54.

16. Morris GM, Huey R, Olson AJ. Using AutoDock for ligand-receptor docking. Curr Protoc Bioinformatics 2008.

17. Ghosh S, Nie A, An J, Huang Z. Structure-based virtual screening of chemical libraries for drug discovery. Curr Opin Chem Biol 2006;10(3):194-202

18. Kitchen DB, Decornez H, Furr JR, Bajorath J. Docking and scoring in virtual screening for drug discovery: Methods and applications. Nat Rev Drug Discov 2004;3(11):935-49.

19. Studio D. Version 3.1. San Diego, CA: Accelrys; 2011.

20. Sakurai N, Kozuka M, Tokuda H, Mukainaka T, Enjo F, Nishino H, et al. Cancer preventive agents. Part 1: Chemopreventive potential of cimigenol, cimigenol-3, 15-dione, and related compounds. Bioorg Med Chem 2005;13(4):1403-8.

21. Wang XF, Zhou QM, Lu YY, Zhang H, Huang S, Su SB. Glycyrrhetinic acid potently suppresses breast cancer invasion and metastasis by impairing the p38 MAPK-AP1 signaling axis. Expert Opin Ther Targets 2015;19(5):577-87.

22. Jiang F, Li Y, Mu J, Hu C, Zhou M, Wang X, et al. Glabridin inhibits cancer stem like properties of human breast cancer cells: An epigenetic regulation of 148a/SMAd2 signaling. Mol Carcinog 2016;55(5):929-40.

23. O'Hara M, Kiefer D, Farrell K, Kemper K. A review of 12 commonly used medicinal herbs. Arch Fam Med 1998;7(6):523-36.

24. Jia L, Zhao Y, Liang XJ. Current evaluation of the millennium phytomedicine - Ginseng (II): Collected chemical entities, modern pharmacology, and clinical applications emanated from traditional 
Chinese medicine. Curr Med Chem 2009;16(22):2924-42.

25. Yun TK, Choi SY. Preventive effect of ginseng intake against various human cancers: A case-control study on 1987 pairs. Cancer Epidemiol Biomarkers Prev 1995;4(4):401-8.

26. Poma P, Notarbartolo M, Labbozzetta M, Maurici A, Carina V, Alaimo A, et al. The antitumor activities of curcumin and of its isoxazole analogue are not affected by multiple gene expression changes in an MDR model of the MCF-7 breast cancer cell line: Analysis of the possible molecular basis. Int J Mol Med 2007;20(3):329.

27. Sandur SK, Pandey MK, Sung B, Ahn KS, Murakami A, Sethi G, et al. Curcumin, demethoxycurcumin, bisdemethoxycurcumin, tetrahydrocurcumin and turmerones differentially regulate anti-inflammatory and anti-proliferative responses through a ROS-independent mechanism. Carcinogenesis 2007;28(8):1765-73.

28. Simon A, Allais DP, Duroux JL, Basly JP, Durand-Fontanier S,
Delage C. Inhibitory effect of curcuminoids on MCF-7 cell proliferation and structure activity relationships. Cancer Lett 1998;129(1):111-6.

29. McKenna DJ, Jones K, Humphrey S, Hughes K. Black cohosh: efficacy, safety, and use in clinical and preclinical applications. Alternative therapies in health and medicine. 2001 May 1;7(3):93.

30. Arias LJ. Drug targeting strategies in cancer treatment: an overview. Mini reviews in medicinal chemistry. 2011 Jan 1;11(1):1-7.

31. Wang XF, Zhou QM, Lu YY, Zhang H, Huang S, Su SB. Glycyrrhetinic acid potently suppresses breast cancer invasion and metastasis by impairing the p38 MAPK-AP1 signaling axis. Expert opinion on therapeutic targets. 2015 May 4;19(5):577-87.

32. Ambesi-Impiombato A, Bernardo D. Computational biology and drug discovery: From single-target to network drugs. Current Bioinformatics. 2006 Jan 1;1(1):3-13. 